\title{
Hepatitis E in Norway: seroprevalence in humans and swine
}

\author{
H. LANGE ${ }^{1,2 * \dagger, ~ J . ~ Ø V E R B ~} \varnothing^{3} \dagger$, K. BORGEN ${ }^{1}$, S. DUDMAN ${ }^{3}$, G. HODDEVIK HOd, $^{3,4}$ \\ A. M. URDAHL ${ }^{5}$, L. VOLD $^{1}$ AND S. K. SJURSETH ${ }^{5}$ \\ ${ }^{1}$ Norwegian Institute of Public Health, Department of Infectious Disease Epidemiology, Oslo, Norway \\ ${ }^{2}$ European Programme for Intervention Epidemiology Training (EPIET), European Centre for Disease \\ Prevention and Control, Stockholm, Sweden \\ ${ }^{3}$ Norwegian Institute of Public Health, Department of Virology, Oslo, Norway \\ ${ }^{4}$ Armed Forces Medical Services, Institute of Microbiology, Oslo, Norway \\ ${ }^{5}$ Norwegian Veterinary Institute, Oslo, Norway
}

Received 19 January 2016; Final revision 18 August 2016; Accepted 31 August 2016; first published online 27 September 2016

\section{SUMMARY}

In Norway, no published data on seroprevalence of hepatitis E virus (HEV) in humans and swine exists. Serum samples from blood donors, veterinarians, swine farm workers and swine were analysed by ELISA to estimate the seroprevalence of HEV in Norway and to investigate the association between direct contact with swine and HEV seroprevalence in humans. The seroprevalence of HEV IgG antibodies was 30\% (24/79) in farm workers, $13 \%$ (21/163) in veterinarians, 14\% (162/1200) in blood donors and 90\% (137/153) in swine. Our results show a high seroprevalence of HEV in humans and swine in Norway. HEV seroprevalence in farm workers and blood donors increased with age, and veterinarians working with swine were twice as likely to be HEV seropositive compared to other veterinarians. High HEV seroprevalence in farm workers and veterinarians working with swine support previous reports suggesting swine as a reservoir for HEV infections in humans in Europe.

Key words: Estimating, hepatitis E, prevalence of disease, serology, zoonoses.

\section{INTRODUCTION}

Until the beginning of the 21st century, hepatitis $\mathrm{E}$ was considered a rare disease in industrialized countries limited to travellers and persons residing in endemic areas in developing countries [1]. Today HEV is considered endemic in many industrialized countries, also in Europe, posing a risk to the growing population of immunocompromised people [2].

\footnotetext{
* Author for correspondence: Ms. H. Lange, Norwegian Institute of Public Health, Department of Infectious Disease Epidemiology, P.O. Box 4404, Nydalen, No-0403 Oslo, Norway.

(Email: Heidi.Lange@fhi.no)

$\dagger$ These authors contributed equally to this work.
}

HEV is synonymous with the species Orthohepevirus $A$ in the genus Orthohepevirus and is the sole member of the family Hepeviridae. It is a single-stranded RNA virus with four known genotypes. Genotypes 1 (HEV-1) and 2 (HEV-2) are restricted to humans, and transmitted faecal-orally via contaminated drinking water in developing countries [2]. Genotypes 3 (HEV-3) and 4 (HEV-4) infect humans, swine and other mammals. Whereas HEV-4 is mainly detected in Southeast Asia, HEV-3 causes sporadic HEV infections worldwide. HEV infections are normally mild and self-limiting [3]; however, in developing countries the case fatality can reach $10-20 \%$ in pregnant women [2]. 
Although the transmission route for HEV-3 is not fully understood, studies have suggested that wild boars and domestic swine are the main reservoirs for human infection [3], either through direct contact or through the consumption of raw or undercooked meat from animals with active infection [4-6]. Additionally, HEV has been isolated from untreated wastewater, swine manure, swine slurry storage facilities, river water and shellfish [3].

HEV seroprevalence in swine ranges from $46 \%$ to $100 \%$ in swine herds and from $23 \%$ to $90 \%$ in individual swine [7-10]. Positive polymerase chain reaction (PCR) indicating active HEV infection have been detected in most investigated swine herds and range from $8 \%$ to $73 \%$ in swine across all age groups [11, 12].

In Norway, HEV has been isolated from sewage [13], but no published data on seroprevalence of HEV in humans or animals exists. The objectives of this study were to estimate the seroprevalence of HEV in humans and swine in Norway, and investigate the association between direct contact with swine and HEV seroprevalence in humans.

\section{METHODS}

\section{Study population}

\section{Blood donors}

A convenience sample of serum from blood donors was collected from 10 different hospitals, located in urban and rural, as well as coastal and inland areas of Norway. Two millilitres of serum from 100 consecutive blood donors was collected at each hospital, except for Oslo which included 200 donors. Serum collected from military recruits, participating in an ongoing seroprevalence study on vaccine-preventable diseases were also included. Information on age and sex was registered, and the serum samples were frozen at $-20^{\circ} \mathrm{C}$ until they were sent to the Norwegian Institute of Public Health (NIPH) for testing.

\section{Veterinarians and swine farm workers}

We used convenience sampling to select veterinarians and farm workers attending two national vocational conferences in 2013. All participants were given written information about HEV and the study, and provided informed consent before having a blood sample taken. The blood was centrifuged and $2 \mathrm{ml}$ serum was stored at $-20^{\circ} \mathrm{C}$ until testing.

\section{Swine}

A large number of blood samples have been systematically collected from Norwegian swine herds since 1994 as part of the national surveillance and control programme for specific viral infections in swine. These samples are stored at $-20^{\circ} \mathrm{C}$. For this study, individual serum samples collected from 153 herds sampled in 1994 (49 herds), 2009 (seven herds) and 2010 (97 herds), were analysed for antibodies against HEV. The number of herds included from each region was proportional to the total number of herds in that region. Additionally, in 2013 a total of 118 swine faecal samples were collected in eight herds representing different parts of Norway. The faecal samples were stored at $-80^{\circ} \mathrm{C}$.

\section{Survey in veterinarians and farm workers}

We developed a standardized self-reporting questionnaire for veterinarians and farm workers attending the above-mentioned conferences. Besides information on age and sex, veterinarians were also asked to state their field of work, i.e. small animal practice, large animal practice, mixed practice or other kind of work experience. Completion of the questionnaire was a prerequisite to drawing blood samples.

\section{Laboratory analyses}

\section{Detection of anti-HEV antibodies}

The National Hepatitis Reference Laboratory at the Department of Virology at NIPH performed testing of all human serum samples. HEV seroprevalence was determined using Wantai anti-HEV IgG ELISA assay kit (Beijing Wantai Biological Pharmacy Enterprise Ltd, China). Positive samples and all samples from farm workers were further tested for anti-HEV IgM using an ELISA assay from Wantai (Beijing Wantai Biological Pharmacy Enterprise Ltd).

The Norwegian Veterinary Institute (NVI) performed testing of all swine serum samples. IgG antibodies against HEV were detected using the ID Screen $^{\circledR}$ Hepatitis E Indirect Multi-species ELISA kit (IDVet, France).

\section{Detection of hepatitis $\mathrm{E}$ virus by real-time reverse transcription-PCR (RT-PCR)}

IgM positive human serum samples

RNA was isolated from $140 \mu \mathrm{l}$ serum using the QIAamp Viral RNA Mini kit (Qiagen, Germany). 
HEV-RNA analyses were performed by RealStar HEV RT-PCR kit 1.0 (Altona Diagnostics GmbH, Germany).

\section{Swine faecal specimens}

Each pool was prepared as a $5 \%$ faecal suspension $(0 \cdot 5 \mathrm{~g}$ faeces in $10 \mathrm{ml} 0.9 \% \mathrm{NaCl}$ ) and centrifuged. RNA was isolated from $140 \mu 1$ of the supernatant using the QIAamp Viral RNA Mini kit (Qiagen). RNA concentration was measured using a NanoDrop 2000 spectrophotometer (Thermo Fisher Scientific, USA). The HepatitisE@ceeramTools kit (Ceeram, France) was used for real-time PCR analysis.

\section{Sequencing of PCR products}

For further characterization and comparison, the open reading frame 2 (ORF2) region of HEV RNA of all PCR-positive specimens was sequenced as previously described [14]. A two-step nested RT-PCR using SuperScript III (Life Technologies, USA) and Platinum Quantitative PCR SuperMix-UDG (Life Technologies) was used for sequencing of the capsid gene. The external primer set HE3156frw and HE3157rev and the internal primer set HE3158frw and HE3159rev were used to amplify the final PCR product of $347 \mathrm{bp}$, which was analysed on agarose gel prior to purification using ExoSap (Affymetrix, USA). The PCR product was sequenced using the internal primer set, the ABI BigDye Terminator v. 3.1 Cycle Sequencing kit and the ABI 3130 Genetic Analyzer (Applied Biosystems, UK). Sequences were assembled using Sequencher v. 4.9 (GeneCodes Corporation, USA).

\section{Statistical analyses}

We described the human study participants by time and person and calculated seroprevalence and prevalence ratios (PRs) with corresponding 95\% confidence intervals (CIs) by age group, sex and work experience. HEV seroprevalence in swine was calculated by year of sampling. All calculations were performed using Stata v. 13 statistical software (StataCorp., USA).

\section{RESULTS}

\section{Blood donors}

A total of 1100 serum samples from blood donors and 100 serum samples from military recruits were collected from January 2013 until March 2013. Information on age and sex were missing for 201 and 203 donors, respectively. The median age was 45 years (range 18-83) and 56\% were male. Anti-HEV IgG was detected in 14\% (162/1200) of the samples and the seroprevalence in males was $59 \%$. Increasing age, but not sex was associated with HEV seroprevalence (Table 1).

Anti-HEV IgM was detected in serum samples from three blood donors, but HEV RNA could only be detected in one of the IgM-positive blood donor samples after real-time PCR analysis. Due to low viral load in the specimen, the PCR product could not be sequenced.

\section{Veterinarians}

A total of 163 veterinarians completed the questionnaire and donated a blood sample. The median age was 43 years (range 24-86) and $32 \%$ were male. Anti-HEV IgG was found in 13\% (21/163) of the samples, and the prevalence in males was $38 \%$. Age and sex was not associated with increased HEV seroprevalence in veterinarians. When stratifying by current work experience, HEV seroprevalence in veterinarians working with swine $(n=46)$ was two times higher compared to those who did not work with swine $(n=117) \quad(\mathrm{PR} 2 \cdot 3,95 \%$ CI 1.0-5.0) (Table 1). Anti-HEV IgM was not found in any of the serum samples from veterinarians.

\section{Farm workers}

A total of 79 swine farm workers completed the questionnaire and donated blood samples. The median age was 51 years (range 23-69) and 59\% were male. Anti-HEV IgG was found in 30\% (24/79) of the samples and the prevalence in males was $67 \%$. HEV seroprevalence increased with age (Table 1). HEV seroprevalence was 2.5 times higher in farm workers compared to blood donors (PR 2·3, 95\% CI 1·6-3.2) and veterinarians (PR $2 \cdot 4,95 \%$ CI $1 \cdot 4-4 \cdot 0$ ). Anti-HEV IgM was found in serum samples from four farm workers, no HEV RNA was detected by real-time PCR analysis.

\section{Swine}

A total of 663 individual swine serum samples from 153 herds were analysed for antibodies against HEV. Anti-HEV IgG was detected in $90 \%$ (137/153) of the herds, and in $73 \%(484 / 663)$ of the individual serum 
Table 1. Number of HEV-positive samples, total number of samples, HEV seroprevalence, prevalence ratios (PR) and $95 \%$ confidence intervals (CI) by age and sex in blood donors, veterinarians and farm workers and by work experience in veterinarians in Norway, 2013

\begin{tabular}{|c|c|c|c|c|c|c|c|c|c|c|c|c|c|c|c|}
\hline & \multicolumn{5}{|c|}{ Blood donors } & \multicolumn{5}{|c|}{ Veterinarians } & \multicolumn{5}{|c|}{ Farm workers } \\
\hline & HEV & Total & $\%$ & PR & $95 \% \mathrm{CI}$ & HEV & Total & $\%$ & PR & $95 \% \mathrm{CI}$ & HEV & Total & $\%$ & PR & $95 \% \mathrm{CI}$ \\
\hline \multicolumn{16}{|l|}{ Age, years } \\
\hline$\leqslant 30$ & 11 & 187 & 6 & & & 1 & 19 & 5 & & & 0 & 6 & & & \\
\hline $31-40$ (ref.: $\leqslant 30)$ & 14 & 167 & 8 & $1 \cdot 4$ & $0 \cdot 7-3 \cdot 1$ & 5 & 46 & 11 & $2 \cdot 1$ & $0 \cdot 3-16 \cdot 5$ & 2 & 10 & 20 & -* $^{*}$ & \\
\hline $41-50$ (ref.: $\leqslant 30)$ & 26 & 304 & 9 & $1 \cdot 5$ & $0 \cdot 7-2 \cdot 9$ & 5 & 47 & 11 & $2 \cdot 0$ & $0 \cdot 3-16 \cdot 2$ & 5 & 22 & 23 & $1 \cdot 8$ & $0 \cdot 4-8 \cdot 2$ \\
\hline $51-60$ (ref.: $\leqslant 30)$ & 54 & 244 & 22 & $3 \cdot 8$ & $2 \cdot 0-7 \cdot 0$ & 9 & 36 & 25 & $4 \cdot 8$ & $0 \cdot 6-34 \cdot 7$ & 14 & 37 & 38 & $3 \cdot 0$ & $0 \cdot 8-11 \cdot 1$ \\
\hline$\geqslant 61($ ref.: $\leqslant 30)$ & 27 & 97 & 28 & $4 \cdot 7$ & $4 \cdot 5-9 \cdot 1$ & 1 & 15 & 7 & $1 \cdot 3$ & $0 \cdot 1-18 \cdot 6$ & 3 & 4 & 75 & $6 \cdot 0$ & $1 \cdot 5-24.9$ \\
\hline$>50($ ref.: $\leqslant 50)$ & 81 & 341 & 24 & $3 \cdot 1$ & $2 \cdot 2-4 \cdot 2$ & 10 & 51 & 20 & $2 \cdot 0$ & $0 \cdot 9-4 \cdot 3$ & 17 & 41 & 41 & $2 \cdot 3$ & $1 \cdot 1-4 \cdot 8$ \\
\hline Unknown & 30 & 201 & 15 & & & & & & & & & & & & \\
\hline \multicolumn{16}{|l|}{ Gender } \\
\hline Male (ref.: female) & 78 & 562 & 14 & $1 \cdot 1$ & $0 \cdot 8-1 \cdot 5$ & 8 & 52 & 15 & $1 \cdot 3$ & $0 \cdot 6-3 \cdot 0$ & 16 & 47 & 34 & $1 \cdot 3$ & $0 \cdot 6-2 \cdot 7$ \\
\hline Female & 54 & 435 & 12 & & & 13 & 111 & 12 & & & 8 & 31 & 26 & & \\
\hline \multicolumn{16}{|l|}{ Swine practice } \\
\hline Yes (ref.: no) & & & & & & 10 & 46 & 22 & $2 \cdot 3$ & $1 \cdot 0-5 \cdot 0$ & & & & & \\
\hline No & & & & & & 11 & 117 & 9 & & & & & & & \\
\hline Total & 162 & 1200 & 14 & & & 21 & 163 & 13 & & & 24 & 79 & 30 & & \\
\hline
\end{tabular}

HEV, Hepatitis E virus.

* Ref.: $\leqslant 40$, no HEV seropositive $\leqslant 30$.

samples. In 1994, 94\% (46/49) of the tested herds were positive compared to $88 \%(91 / 104)$ in 2009/2010.

Of the eight herds sampled, HEV RNA was detected in faecal samples from three herds. The samples were sequenced, and alignments with published strains showed the Norwegian HEV isolates to be HEV-3. The sequences have been submitted to GeneBank under accession number KU892216-19.

\section{DISCUSSION}

This study shows that HEV is endemic in swine in Norway, and that the virus has been endemic in Norwegian swine herds for at least 20 years. Our findings indicate that swine represents a HEV reservoir for human infection, and that direct contact with swine is associated with an increased risk of HEV infection. Farm workers and veterinarians working with swine were more than twice as likely to be HEV seropositive compared to blood donors and veterinarians not working with swine. An increased risk of HEV infection in people with direct contact with swine has also been reported in crosssectional, seroprevalence studies from other countries $[6,15]$. The seroprevalence of HEV in farm workers and blood donors increased with age and those aged $>50$ years were $2-3$ times more likely to be HEV positive compared to those aged $\leqslant 50$ years. This confirms previously reported findings [3], and may be caused by cumulative lifetime exposure to the virus. However, a decline in the number of $\mathrm{HEV}$ IgG-positive persons has been reported in The Netherlands and Denmark over the last decades [5, 16] indicating a lowered infectious pressure and thereby less seropositive young people. Although not statistically significant, the observed decrease in seropositive swine from 1994 to 2009/2010 may have caused a lowered human infectious pressure also in Norway. As HEV can be transmitted through contaminated raw or undercooked swine products [17, 18], application of basic hygienic measures in the kitchen, and ensuring sufficient heating of meat products can help prevent the chain of swine to human transmission in the overall population.

The Wantai IgG assay used to analyse the human serum samples in this study has a higher sensitivity compared to most other commercial assays $[19,20]$ without any loss of specificity [21, 22]. Compared to studies from other European countries using the same ELISA kit as the present study, the seroprevalence of HEV IgG in Norwegian blood donors is lower than in The Netherlands (27\%) and France (23.6\%), comparable to results in blood donors from Upper Austria (13.6\%) and England (12\%), but higher than 
in Scotland (4.7\%) [23-25]. The reason for this large variation between countries may stem from differences in study populations and incidental variations between small studies; although it appears that some of these differences are true geographical variations. The high HEV seroprevalence in Norwegian blood donors as reported in this study makes autochthonous zoonotic transmission likely. Screening of blood products for HEV is currently not routine practice in Norwegian hospitals, but the detection of anti-HEV IgM and HEV RNA in some of the blood donor serum samples in this study implies that transmission of HEV via blood transfusion is possible. Immunocompromised patients, in particular the solid organ transplant patients, are at high risk for developing chronic HEV infection through HEV-infected blood products [26]. Therefore, performance of a risk assessment should be considered to decide if screening of blood products should be done before use in vulnerable patient groups. So far, there have been no reported cases of HEV infection in immunocompromised patients in Norway, but this may relate to the lack of routine testing for HEV in these patients.

This cross-sectional study is limited by the fact that it is carried out at a single time point and provides no indication of the sequence of events, i.e. whether an exposure occurred before, after or during the onset of the HEV infection. In addition, the use of convenience sampling precluded the extrapolation of the seroprevalence measured in farm workers, veterinarians or blood donors in general. The veterinarians included in this study were mostly veterinarians with less exposure to swine, due to a concurrent conference on swine practice at the time of sampling. This may have led to an underestimation of the association found between direct contact with swine and HEV seropositivity. In addition, we have no information regarding direct contact with swine from the blood donors included in the study, possibly resulting in a further underestimation of this association.

In conclusion, this study supports the theory of a HEV reservoir in the Norwegian swine population with a potential zoonotic transmission to humans. Based on our findings, we recommend increased testing for HEV in patients with acute hepatitis and in immunocompromised patients with elevated liver enzymes. Furthermore, the association between direct contact with swine and the increased risk of HEV infection emphasizes that veterinarians and farm workers should take appropriate precautions to reduce the risk of zoonotic transmission of HEV.

\section{ACKNOWLEDGEMENTS}

The authors acknowledge all the blood transfusion services, transfusion medicine departments and Institute of Microbiology, Armed Forces Medical Services, Norway, who collected all the human blood samples, the veterinarians and Animalia who collected samples from swine, and scientific staff in the two laboratories responsible for diagnostic analyses of the human and swine samples. Special thanks go to Bjørn Lium, Adam Zerihun and Anna Germundsson Hauge from the Norwegian Veterinary Institute for their support in the planning and conduct of this work, and to Sissel Orstein Wiik from the Norwegian Institute of Public Health for her work in the implementation of the serological methods. In addition, we also thank Emily MacDonald from the Norwegian Institute of Public Health and Alicia Barrasa and Pawel Stefanoff, scientific coordinators from EPIET, for their useful feedback on the manuscript.

This study was funded by the Norwegian Institute of Public Health, The Norwegian Veterinary Institute and Animalia.

\section{DECLARATION OF INTEREST}

None.

\section{REFERENCES}

1. Zuckerman AJ. Hepatitis E virus. British Medical Journal (Clinical Research Edition) 1990; 300: 14751476.

2. Kamar N, et al. Hepatitis E. Lancet 2012; 379: 24772488.

3. Kamar N, et al. Hepatitis E virus infection. Clinical Microbiology Reviews 2014; 27: 116-138.

4. Van der Poel WH. Food and environmental routes of Hepatitis E virus transmission. Current Opinion in Virology 2014; 4: 91-96.

5. Christensen PB, et al. Time trend of the prevalence of hepatitis $\mathrm{E}$ antibodies among farmers and blood donors: a potential zoonosis in Denmark. Clinical Infectious Diseases 2008; 47: 1026-1031.

6. Krumbholz A, et al. Prevalence of hepatitis E virusspecific antibodies in humans with occupational exposure to pigs. Medical Microbiology and Immunology 2012; 201: 239-244.

7. Yugo DM, Meng XJ. Hepatitis E virus: foodborne, waterborne and zoonotic transmission. International Journal of Environmental Research and Public Health 2013; 10: 4507-4533.

8. Pavio N, Meng XJ, Renou C. Zoonotic hepatitis E: animal reservoirs and emerging risks. Veterinary Research 2010; 41: 46. 
9. Krumbholz A, et al. Age-related and regional differences in the prevalence of hepatitis $\mathrm{E}$ virus-specific antibodies in pigs in Germany. Veterinary Microbiology 2013; 167: 394-402.

10. Ponterio E, et al. Detection of serum antibodies to hepatitis $\mathrm{E}$ virus in domestic pigs in Italy using a recombinant swine HEV capsid protein. BMC Veterinary Research 2014; 10: 133.

11. Berto A, et al. Prevalence and transmission of hepatitis $\mathrm{E}$ virus in domestic swine populations in different European countries. BMC Research Notes 2012; 5: 190.

12. Breum SO, et al. Hepatitis E virus is highly prevalent in the Danish pig population. Veterinary Microbiology 2010; 146: 144-149.

13. Myrmel M, Lange H, Rimstad E. A 1-year quantitative survey of noro-, adeno-, human boca-, and hepatitis e viruses in raw and secondarily treated sewage from two plants in Norway. Food and Environmental Virology 2015; 7: 213-223.

14. Meng XJ, et al. A novel virus in swine is closely related to the human hepatitis E virus. Proceedings of the National Academy of Sciences USA 1997; 94: 9860-9865.

15. Chaussade H, et al. Hepatitis E virus seroprevalence and risk factors for individuals in working contact with animals. Journal of clinical virology 2013; 58: 504-508.

16. Hogema BM, et al. Past and present of hepatitis $E$ in the Netherlands. Transfusion 2014; 54: 3092-3096.

17. Berto A, et al. Hepatitis E virus in pork food chain, United Kingdom, 2009-2010. Emerging Infectious Diseases 2012; 18: 1358-1360.
18. Di Bartolo I, et al. Detection of hepatitis E virus in pork liver sausages. International Journal of Food Microbiology 2015; 193: 29-33.

19. Mansuy JM, et al. Hepatitis E virus antibodies in blood donors, France. Emerging Infectious Diseases 2011; 17: 2309-2312.

20. Bendall R, et al. A comparison of two commercially available anti-HEV IgG kits and a re-evaluation of anti-HEV IgG seroprevalence data in developed countries. Journal of Medical Virology 2010; 82: 799-805.

21. Abravanel F, et al. Performance of anti-HEV assays for diagnosing acute hepatitis $\mathrm{E}$ in immunocompromised patients. Journal of Clinical Virology 2013; 58: 624-628.

22. Pas SD, et al. Diagnostic performance of selected commercial HEV IgM and IgG ELISAs for immunocompromised and immunocompetent patients. Journal of Clinical Virology 2013; 58: 629-634.

23. Gallian $\mathbf{P}$, et al. Hepatitis $E$ virus infections in blood donors, France. Emerging Infectious Diseases 2014; 20: 1914-1917.

24. Fischer C, et al. Seroprevalence and Incidence of hepatitis E in Blood Donors in Upper Austria. PLoS ONE 2015; 10: e0119576.

25. Dalton HR, et al. Hepatitis e virus: current concepts and future perspectives. Current Infectious Disease Reports 2014; 16: 399.

26. Pawlotsky J-M. Hepatitis E screening for blood donations: an urgent need? Lancet 2014; 384: 1729-1730. 